\title{
Comparison between SRK/T and Haigis Formulas on Visual Acuity of Patient with Senile Cataract Post-Phacoemulsification
}

\author{
Delfi Delfi*iD, Vanda Virgayanti (D), Hendra Gunawan \\ Department of Ophthalmology, Faculty of Medicine, Sumatera Utara University, Medan, Indonesia
}

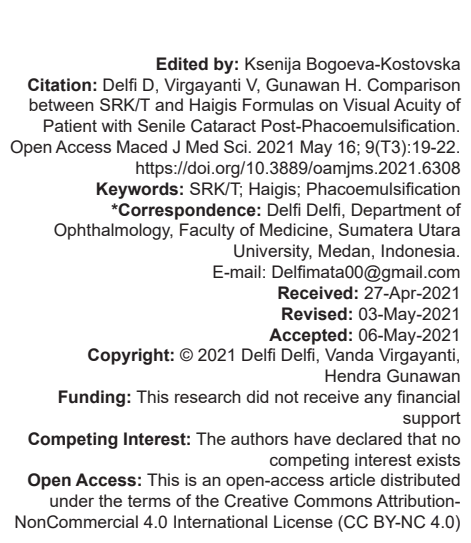

\section{Introduction}

Cataract contributes to the most common cause of blindness worldwide. Cataract is a condition of abnormality in the eye lens in the form of cloudiness or turbidity, resulting in a sharp decrease in vision that occurs in the lens, which can be caused by hydration or protein denaturation, presenting a cloudy or a white image. Cataract is more common to appear to older age [1], [2], [3].

Cataract surgery is one of the most commonly performed procedures throughout the world. With sophisticated and modern technologies, surgical techniques improvements, as well as patient contentment, are essential to determine the outcome result of this procedure. To gain optimal results, preoperative biometry must be precised and the use of a formula to measure the strength of the intraocular lens (IOL) accurately must be applied [4].

The outcome of optimal cataract surgery is influenced by several things in calculating the strength of the IOL, including biometric data before cataract surgery, as well as axial length ( $A L)$, depth of the front chamber anterior chamber depth (ACD), lens thickness, and corneal curvature. The formula for calculating the strength of the $\mathrm{IOL}$ and the quality of the IOL is also another important aspect of biometric measurements before cataract surgery. The accuracy of biometric examinations is very useful in cataract surgery to minimize refractive errors and good visual acuity results. After surgery, it is expected that the target of sharp visual emetropia is achieved without using glasses or contact lenses [5], [6].

Calculation of the IOL formula has evolved since 1949 when Harold Ridley implanted the first $\mathrm{IOL}$ in the human eye. There are various theories and regressions formulas available for the calculation of IOL strength. These consist of Holladay 1, Hoffer Q, and SRK-T (known as the third-generation formula) and Holladay 2, Haigis, and Olsen as the fourth-generation formula or the latest [7], [8], [9]. A study conducted by Wang et al. in China on SRK/T, showed that SRK/T is the most accurate third-generation formula, using IOL Master data with SRK/T produces the most precise refraction [10].

One of the factors that contributed to the accuracy of the IOL calculation was the accuracy of the biometric data to be applied in the IOL strength calculation formula. Based on the background above, the researcher is interested in conducting a study on the comparison between the changes in visual acuity in 
post-phacoemulsification patients using the SRK/T and Haigis formulas.

\section{Methods}

This study was an observational prospective analytic series by taking data at eye polyclinic patients at Medan Baru Eye Hospital from June 2019 to August 2019. Data were taken from the subject group divided into two groups using both variables.

The inclusion criteria for this study were all $\geq 40$ years older cataract patients who would undergo phacoemulsification, willing to be sampled and for follow-up after the $7^{\text {th }}$ and $30^{\text {th }}$ days of operation. Exclusion criteria for this study were patients who experienced intraoperative and post-operative complications, anterior segment abnormalities, systemic abnormalities, and non-follow-up.

Samples were taken by measuring $A L$, the depth of anterior chamber, corneal curvature, and IOL (using biometric IOL Master 500) using the SRK/T or Haigis formula. The strength of the IOL was chosen based on the post-operative refraction prediction that was closest to Plano. On days 7 and 30 postoperatively, patients were followed up and visual acuity was tested with Snellen charts. The best correction results were checked 1 month after surgery and the spherical equivalent was then calculated. Furthermore, the calculation of mean error (ME) and the percentages in $\pm 0.25 \mathrm{D}, \pm 1.00$, and $\pm 1.50 \mathrm{D}$ and calculating the p-value based on each group of formulas. The data were processed using the Mann-Whitney U-test to see accuracy of using the SRK/T and Haigis formulas.

\section{Results}

This research was an analytical prospective study. The total number of research subjects was 122 patients (122 eyes), where 84 patients (84 eyes) were taken, of which 38 patients did not come back for control on schedule.

Table 1 explains that the average value of the largest gender distribution in the SRK/T formula group was female, namely, 24 eyes (55.8\%). Meanwhile, in the Haigis formula group, the highest sex was male as many as 23 eyes (56.1\%). For the average $A L$, it was divided into three groups, which were short $(<23)$, medium (23-24), and long (>24) AL.

Characteristics based on age, corneal curvature, and ACDs of patients who would be subjected to undergo phacoemulsification procedure are based
Table 1: Characteristics based on gender and $A L$ in patients who will undergo phacoemulsification

\begin{tabular}{lll}
\hline Characteristics & SRK/T n (\%) & Haigis n (\%) \\
\hline Gender & $18(43.9)$ & $23(56.1)$ \\
$\quad$ Male & $24(55.8)$ & $19(44.2)$ \\
$\quad$ Female & $13(72.2)$ & $5(27.8)$ \\
AL & $26(57.8)$ & $19(42.2)$ \\
$\quad$ Short $(<23)$ & $3(14.3)$ & $18(85.7)$ \\
$\quad$ Medium (23-24) & & \\
$\quad$ Long (>24) & &
\end{tabular}

on Table 2. It showed the data of each group totaling of 42 study subjects.

Table 2: Characteristics based on age, corneal curvature, and $A C D$ in patients who will undergo phacoemulsification

\begin{tabular}{lllll}
\hline Characteristics & $\mathrm{n}$ & SRK/T $($ Mean \pm SD) & $\mathrm{n}$ & Haigis (Mean \pm SD) \\
\hline Age & 42 & $64.19 \pm 10.61$ & 42 & $61.98 \pm 9.35$ \\
$\mathrm{~K}_{1}$ & 42 & $43.63 \pm 1.32$ & 42 & $43.28 \pm 1.31$ \\
$\mathrm{~K}_{2}$ & 42 & $44.66 \pm 1.18$ & 42 & $44.10 \pm 1.34$ \\
ACD & 42 & $3.27 \pm 0.21$ & 42 & $3.44 \pm 0.26$ \\
\hline ACD: Anterior chamber depth. & & &
\end{tabular}

Based on Table 3, it showed changes in visual acuity seen on the $7^{\text {th }}$ day after phacoemulsification, where the best visual acuity $(6 / 6-6 / 18)$ was found in the Haigis formula group, namely, 40 eyes $(51.3 \%)$ compared to the SRK/T formula group, as many as 38 eyes $(48.7 \%)$. There was a visible change in visual acuity on the $30^{\text {th }}$ day after phacoemulsification, where the most visible change was found in the best visual acuity 6/6-6/18 in the Haigis formula group of 42 eyes $(51.2 \%)$ compared to the SRK/T formula group of 40 eyes $(48.8 \%)$.

Table 3: Comparison of changes in visual acuity on day +7 and day +30 post-phacoemulsification using SRK/T and Haigis formulas

\begin{tabular}{llllll}
\hline Visual acuity & Day +7 & & & \\
\cline { 2 - 3 } & SRK/T n $(\%)$ & Haigis $\mathrm{n}(\%)$ & & SRK/T $\mathrm{n}(\%)$ & Haigis $\mathrm{n}(\%)$ \\
\hline $6 / 6-6 / 18$ & $38(48.7)$ & $40(51.3)$ & & $40(48.8)$ & $42(51.2)$ \\
$<6 / 18-6 / 60$ & $4(66.7)$ & $2(33.3)$ & & $2(100)$ & 0 \\
\hline
\end{tabular}

Table 4 shows the comparison of the accuracy of the predicted results/ME of post-phacoemulsification using the SRK/T formula and Haigis, using the MannWhitney U-test, it resulted in no significant difference between the SRK/T and Haigis formulas in predicting the accuracy of the ME results.

Table 4: Comparison between result prediction (ME) accuracy of post-phacoemulsification refraction using SRK/T and Haigis formulas

\begin{tabular}{llll}
\hline Prediction (ME) & \multicolumn{1}{l}{ Formula } & $\mathrm{p}$ \\
\cline { 2 - 3 } & SRK/T n (\%) & Haigis $\mathrm{n}(\%)$ & \\
\hline $\pm 0.25 \mathrm{D}$ & $39(48.8)$ & $41(51.3)$ & 0.053 \\
$\pm 1.00 \mathrm{D}$ & $40(50)$ & $42(100)$ & \\
$\pm 1.50 \mathrm{D}$ & $42(100)$ & 0 & \\
\hline${ }^{*}$ Mann-Whitney U-test, ME: Mean Error & & &
\end{tabular}

Table 5 shows the comparison of the accuracy of the ME prediction using the SRK/T formula and Haigis with axis length group.

Based on Table 6 , the prediction of residual refractive errors after phacoemulsification using the $\mathrm{SRK} / \mathrm{T}$ and Haigis formulas was illustrated, where the most myopia refractive errors were found in the SRK/T formula, which were 3 eyes $(75.0 \%)$ compared to the Haigis formula which was only 1 eye $(25.0) \%)$. The 
Table 5: Comparison between result prediction (ME) accuracy of post-phacoemulsification refraction using SRK/T and Haigis formulas toward $\mathrm{AL}$

\begin{tabular}{llll}
\hline AL & Prediction (ME) & Formula & \\
\cline { 3 - 4 } & & SRK/T n (\%) & Haigis n (\%) \\
\hline Short & $\pm 0.25 \mathrm{D}$ & $11(68.8)$ & $5(31.3)$ \\
& $\pm 1.00 \mathrm{D}$ & $12(100)$ & 0 \\
\multirow{4}{*}{ Medium } & $\pm 1.50 \mathrm{D}$ & $13(100)$ & 0 \\
& $\pm 0.25 \mathrm{D}$ & $25(56.8)$ & $19(43.2)$ \\
\multirow{2}{*}{ Long } & $\pm 1.50 \mathrm{D}$ & $26(100)$ & 0 \\
& $\pm 0.25 \mathrm{D}$ & $3(15.0)$ & $17(85.0)$ \\
& $\pm 1.00 \mathrm{D}$ & 0 & $18(100)$ \\
\hline AL: Axial length, ME: Mean error. & &
\end{tabular}

prediction results for emetropia were mostly found in the Haigis formula with 41 eyes (51.3\%) compared to the SRK/T formula with 39 eyes $(48.8 \%)$.

Table 6: Overview of post-phacoemulsification refraction abnormalities remains using SRK/T and Haigis formulas

\begin{tabular}{lll}
\hline Refraction prediction & SRK/T n (\%) & Haigis $\mathrm{n}(\%)$ \\
\hline Myopia & $3(75.0)$ & $1(25.0)$ \\
Emetropia & $39(48.8)$ & $41(51.3)$ \\
\hline
\end{tabular}

\section{Discussion}

The cataract surgery performed in this study was phacoemulsification, where the goal of the outcome of this procedure was an optimal postoperative refraction. In conducting this research, the researcher attempted to measure keratometry accurately, to select the operating technique, the right power IOL strength, and the IOL strength calculation formula. It is expected that the examination using the IOL Master biometry can achieve the right results and be helpful in producing optimal post-operative refraction.

In this study, it was found that the highest average age in the SRK/T formula group was $64.19 \pm$ 10.61 while in the Haigis formula group was $61.98 \pm$ 9.35. This is in accordance with a study conducted by Nemeth et al. where the average age of the research subjects was $70.5 \pm 11.0$ years, all cataract patients in this study were senile cataracts, so that, the age of the research subjects was more than 40 years old, this is considering senile cataracts are the most common type of cataract [11].

The characteristics of the average ACD appear to be longer on Haigis formula $(3.44 \pm 0.26)$, than SRK/T formula group $(3.27 \pm 0.21)$. These conditions are affected by racial factors and geographical structure that influences the variations of the eye anatomy [12].

The accuracy of the prediction of the ME of post-phacoemulsification refraction using the SRK/T formula and Haigis formula, which can be seen that the percentage of ME from the Haigis formula was reached at its maximum of $100 \%$ at ME $\pm 1.00 \mathrm{D}$, while the SRK/T formula was reached at a maximum of $\pm 1.50 \mathrm{D}$. Based on the guidelines from the Royal College of Ophthalmologist (RCOpth) determined that $97 \%$ of patients should achieve results of a least $97 \%$ refraction within $\pm 1.00 \mathrm{D}$ from the predicted value obtained [13].

Using the Mann-Whitney U-test, it was found that there was no significant difference between the SRK/T and Haigis formulas in predicting the accuracy result of the ME. This is in line with research conducted by Faramarzi et al. which stated that in eyes with steep corneas, statistically, it showed no significant difference between the accuracy of the four formulas (Haigis, Holladay 1, Hofer-Q, SRK/T), and general IOL power calculations [14].

This is not in line with the research conducted by Sharma et al. where the research compared the prediction accuracy of the SRK/T and Haigis formulas without optimized constants where the SRK/T formula was in $\pm 1.00 \mathrm{D}$ and $\pm 2.00 \mathrm{D}$ which were $78 \%$ and $96 \%$, while in the Haigis formula, without personal constants within $\pm 1.00 \mathrm{D}$ and $\pm 2.00 \mathrm{D}$ were $86 \%$ and $100 \%$, where the Haigis formula was significantly more precised than the SRK/T formula [15]. Mansour et al. (2018) explained that the Haigis formula was more precised than the SRK/T formula in predicting postoperative outcome [16].

This study also described the prediction of residual refractive errors after phacoemulsification using the SRK/T and Haigis formulas, where it was detected that myopia refractive errors were mostly found in the SRK/T formula, which were discovered in 3 eyes $(75.0 \%)$ compared to the Haigis formula, which was only 1 eye $(25.0 \%)$. The most emetropia was found in the Haigis formula with 41 eyes $(51.3 \%)$ compared to the SRK/T formula, which involved 39 eyes $(48.8 \%)$. These results were corresponded with previous research performed by Karabela et al. (2016) in Turkey on the outcome of the SRK/T formula using A-scan ultrasound biometry after a phacoemulsification procedure, explaining that the SRK/T formula had a slight tendency to become myopia [17].

\section{Conclusions}

The characteristics of the subjects found in this study based on gender, showed that more female patients were included in the SRK/T formula group. Based on age, the oldest subject was found in the $\mathrm{SRK} / \mathrm{T}$ formula group. From the biometric data, it can be seen that the depth of the front eye chamber (ACD) was deeper in the Haigis formula group, and K1 and $\mathrm{K} 2$ in the biometric data appeared to be higher in the Haigis formula group.

Prediction of the accuracy of visual acuity changed after phacoemulsification using the SRK/T formula to reach $100 \%$ over the $\pm 1.50 \mathrm{D}$ limit was not achieved according to post-operative target for cataract removal of $\pm 1.00 \mathrm{D}$. 
Prediction of the accuracy of changes in visual acuity after phacoemulsification using the Haigis formula without personal constant showed that the formula to reach $100 \%$ was finally reached at \pm 1.00 $\mathrm{D}$ limit, this was in accordance with the post-operative target of cataract extraction of $\pm 1.00 \mathrm{D}$, but there was no statistically significant difference.

\section{References}

1. Alshamrani AZ. Cataracts pathophysiology and managements. Egypt J Hosp Med. 2018;70(1):151-4.

2. Michael R, Born AJ. The aging lens and cataract: A model normal and pathological ageing. Philos Trans $\mathrm{R}$ Soc. 2011;366(1568):1278-92.

PMid:21402586

3. Gupta VB, Rajagopala M, Ravishankar B. Ethiopathogenesis of cataract: An appraisal. Indian J Ophthalmol. 2014;62(2):103-10. https://doi.org/10.4103/0301-4738.121141 PMid:24618482

4. Hope-Ross M, Mooney D. Intraocular lens power calculation. Eye (Lond). 1988;2(4):367-9. https://doi.org/10.1038/eye.1988.67 PMid:3253128

5. Farag MM, Atwa FA. Haigis and SRK/T formulas for intraocular lens power calculations in high myopia. Menoufiya Med $\mathrm{J}$ 2011;24(1):155-60.

6. Drexler W, Findl O, Menapace R, Rainer G, Vass C Hitzenberger CK, et al. Partial coherence interferometry: A novel approach to biometry in cataract surgery. Am J Ophthalmol. 1998;126(4):524-34. https://doi.org/10.1016/ s0002-9394(98)00113-5

PMid:9780097

7. MacLaren RE. Biometry accuracy using zero and negative powered intraocular lenses. J Cataract Refract Surg. 2005;31(2):280-90. https://doi.org/10.1016/j.jcrs.2004.04.054 PMid: 15767147

8. Nafees RE. Intraocular lens power calculation in patients with high axial myopia before cataract surgery. Saudi J Ophthalmol. 2010;24:77-80. https://doi.org/10.1016/j.sjopt.2010.03.006
9. Olsen T. Improved accuracy of intraocular lens power calculation with the Zeiss IOL Master. Acta Ophthalmol Scan. 2007;85(1):847. https://doi.org/10.1111/j.1600-0420.2006.00774.x PMid:17244216

10. Wang JK, Hu CY, Chang SW. Intraocular lens power calculation using the IOL master and various formulas in eyes with long axial length. J Cataract Refract Surg. 2008;34(2):262-7. https:// doi.org/10.1016/j.jcrs.2007.10.017

PMid:18242451

11. Nemeth G, Nagy A, Berta A, Modis L. Comparison of intraocular lens power predicting uisng immersion ultrasound and optical biometry with and without formula optimizing. Graefes Arch Clin Exp Ophthalmol. 2012;250(9):1321-5. https://doi.org/10.1007/ s00417-012-2013-9

PMid:22527318

12. Miraftab $M$, Hahemi $H$, Fotouhi A. Effect of anterior chamber depth on the choice of intraocular lens calculation formula in patients with normal axial length. Middle East Afr J Ophthalmol. 2014;21(4):307-11. https://doi.org/10.4103/0974-9233.142266 PMid:25371635

13. Gale RP, Saldana M, Jhonston RL, Zuberbuhler B, McKibbin M. Benchmark standards for refractive outcomes after NHS cataract surgery. Eye. 2009;23(1):149-52. https://doi. org/10.1038/sj.eye.6702954

PMid: 17721503

14. Faramarzi A, Aghajani A, Ghiasian L. Accuracy of various intraocular lens power calculation formulas in steep corneas. $J$ Ophthalmic Vis Res. 2017;12(4):385-9. https://doi.org/10.4103/ jovr.jovr_20_17 PMid:29090047

15. Sharma R, Maharajan P, Kotta S. Prediction of refractive outcome after cataract surgery using partial coherence interferometry: Comparison of SRK/T and Haigis formula. Int Ophthalmol. 2014;34(3):451-5. https://doi.org/10.1007/s10792-012-9671-9 PMid:24682595

16. Mansour MN, Kamel RM, Hegazy HS. Comparison of SRK/T and Haigis formula in the predizction of refractive outcome after phacoemulsification. Sci J Al-Azhar Med Facult. 2018;2(2):85-9. https://doi.org/10.4103/sjamf.sjamf_21_18

17. Karabela $Y$, Eliacik M, Kaya F. Performance of SRK/T formula using A-Scan ultrasound biometry after Phacoemulsification in eye with short and long axial lengths. BMC Ophthalmol. 2016;16:96. https://doi.org/10.1186/s12886-016-0271-8 